\title{
Teams Frightened of Failure Fail More: Modelling Reward Sensitivity in Teamwork
}

\author{
Siyuan Guo \\ Department of Computer Science, UCL \\ London, United Kingdom \\ siyuan.guo96@outlook.com
}

\author{
Soo Ling Lim \\ Department of Computer Science, UCL \\ London, United Kingdom \\ s.lim@cs.ucl.ac.uk
}

\author{
Peter J. Bentley \\ Department of Computer Science, UCL \\ London, United Kingdom \\ p.bentley@cs.ucl.ac.uk
}

\begin{abstract}
According to Gray's Reinforcement Sensitivity Theory (RST), individuals have differing sensitivities to rewards and punishments, which in turn affect their behaviours. The behavioural inhibition system (BIS) is associated with sensitivity to punishment while the behavioural activation system (BAS) is associated with sensitivity to reward. In this work, we model BIS/BAS by supplementing an existing agent-based model of team collaboration in order to explore the combined effect on team performance for a more complex and realistic personality structure. We investigate the significance of BIS/BAS on team behaviour for tasks with differing levels of uncertainty. Findings include a prediction that for tasks with uncertainty, a majority of personality types are significantly influenced by behavioural activation system, and that all personality types are significantly negatively influenced by behavioural inhibition system. The more sensitive to punishments, the worse teams perform.
\end{abstract}

Keywords-agent-based modelling, collaboration, teamwork, personality psychology, behavioural inhibition system, behavioural activation system

\section{INTRODUCTION}

Feedback forms the basis of learning. Positive and negative reinforcement helps us understand whether we have learned correctly or incorrectly. Our experiences help us to react with different sensitivities to such feedback, helping us to adapt to our varied environments. But this difference is not only caused by our different backgrounds, it is also a psychological trait the sensitivity to rewards and punishment $[\underline{1}, \underline{2}]$ that underpins our behaviours and emotions in different situations. Proposed as a trait with a clear physiological basis, Gray's reinforcement sensitivity theory $[\underline{1}, \underline{2}]$ provides a more grounded view of personality traits such as introversion and neuroticism.

Understanding reward sensitivity in an individual for a relatively well-defined environment can provide us with insights into the likely behaviour of that individual. However, it is considerably more challenging to understand the effects of reward sensitivity within a team, and even more so for uncertain environments. Unfortunately, today's world is full of uncertainties, caused by rapid technological change, pandemics, political upheavals, and climate change. Team collaboration is one of the foundations of human society, so it is important to understand how different people react to reward/punishment cues that affect their teamwork performance. This work investigates this problem through an agent-based model that incorporates reinforcement sensitivity theory.
In previous work, Lim and Bentley [3] developed an agentbased model to investigate how different personalities affect team performance when the shared optimisation task has different levels of noise. They further investigated the effect of personality on conformity [4], the effect of different personality combinations and dynamically changing optimisation task for team performance combining this model with genetic algorithm $[\underline{5}, \underline{6}]$. The model in previous work was based on Jung's Type Theory [7] of extraversion and introversion which are distinguished by their focus on the external or internal world. In this work, we further develop the model to incorporate Gray's reinforcement sensitivity theory [2] which suggests extraversion vs introversion can be viewed as effects of sensitivity to environmental stimuli. We focus on a key question: is reward sensitivity significant compared to the effects of personalities on team performance for uncertain tasks?

The motivation behind this work is to address some issues in psychology research. Human experimentation can create ethical issues and has been increasingly difficult to conduct, making it more difficult to progress our understanding in the area. Our model, by incorporating Gray's reinforcement sensitivity theory, with the computational model developed by Lim and Bentley [3] provides a virtual laboratory to conduct such experiments. In traditional psychological research, BAS/BIS (being sensitive to reward/punishment) has demonstrated a highly variable relationship with team performance: some studies report no evidence for BIS related to performance [ $\underline{8}, \underline{9}]$, others find BIS has significant relationship with performance [10]. Here we resolve this inconsistency: our model predicts that for all personality teams, the more sensitive to punishment, the worse teams perform.

This paper is organised as follows. Section II describes related work. Section III describes the model, Section IV describes the experiments, Section V discusses the results, Section VI discusses the relationship between our simulated predictions and related literature, Section VII concludes.

\section{BACKGROUND}

Personality Traits Research is a field motivated to understand how personality developed and how it influences our behaviours. A trait is a stable characteristic in personality that influences our behaviours and emotions for a long time period and is hard to change once developed. To this end, there are many frameworks developed in attempt to capture the general categories that make up our personality. Some of the 
best-known research include the five-factor model (FFM) [11] where an individual is characterised on five dimensions: openness to experience, conscientiousness, extraversion, agreeableness, and neuroticism. In type theory, personalities are classified into distinct types (e.g., Introverted or Extraverted) rather than measured on a continuum (e.g. the level of extraversion). Some of the best-known work in type theory include Jung's Type Theory [7], and the Myers-Briggs Type Indicator (MBTI), which was built on Jung's work [12] and classifies individuals based on their attitude (Extraverted vs. Introverted), perception (Sensing vs. Intuition) and judgment (Thinking vs. Feeling) ${ }^{1}$. On another dimension, there is research investigating the biological connections between personality and physiological mechanisms. The classic example is Eysenck's three dimensions of personality: Extraversion vs. Introversion, Neuroticism vs. Emotional Stability, Psychoticism vs Self-Control [13]. This has received wide notice and in particular Gray proposed his original Reinforcement Sensitivity Theory (RST) where he introduced the concept of behavioural inhibition system (BIS) and behavioural activation system (BAS) as a modification to Eysenck's Theory [1].

Below we provide (A) an overview of the use of agentbased modelling for human behaviour focusing on the effects of personalities and also (B) a more detailed introduction of the Gray's Theory.

\section{A. Agent-based Models of Personality in Collaboration}

Salvit and Sklar [14] used MBTI to model termites gathering food. In the termite model, Sensing agents focus on food that is close by and return to the place they last saw food if they cannot see food, while Intuitive agents prefer bigger clusters of food and explore new areas when they cannot see food. Thinking agents go straight for their targeted food, while Feeling agents avoid food that their neighbours are targeting. Campos et al. [15] simulated a firefighting scenario where a building is on fire and a person is in danger. Firefighter agents with different MBTI personality types can either put a safety-net in place for the person to jump on it or enter the building to bring the person out. They found that Sensing agents prefer to use the safety-net and Intuitive agents prefer to enter the building. Ahrndt et al. [16] used the five-factor model (FFM) to model ants in a colony working together to collect food and defend themselves. In the ant colony model, agreeableness and extraversion influence an agent's preference to commit to selfish or altruistic goals, and variations on conscientiousness influence an agent's preference to change their intentions. Durupinar [17] extended the High-Density Autonomous Crowds system using the FFM. They provided each agent with personalities that are associated with an existing behaviour in the system and found that specifying an agent's personality leads to an automation of low-level parameter tuning. In their model, people with low conscientiousness and agreeableness cause congestion and neurotic people display panic behaviour.

\section{B. Behavioural Inhibition System (BIS) and Behavioural Activation System (BAS)}

Gray proposes two fundamental orthogonal dimensions of neurological systems that govern the sensitivities of individuals to environmental cues. One of the systems is called behavioural inhibition system (BIS). According to Gray, it is sensitive to signals of punishment, non-reward and novelty. It also prevents behaviours that may lead to negative or painful outcomes. During the development of the questionnaires for BAS/BIS scales, Carver and White [18] characterises BIS as regulation for aversive motivation. The other physiological mechanism is called the behavioural approach system or behavioural activation system (BAS). It is believed to control appetitive motivation. In contrast to BIS, it is sensitive to signals of reward, non-punishment and escape from punishment. A person, being activated in BAS system, will move towards the goal. Furthermore, because BAS and BIS represent distinct structures in the nervous system, their sensitivities are presumed to be orthogonal. Thus, any person can have BIS and BAS sensitivities in any combinations. However, under environmental cues, only one system can be activated at a time [19].

\section{ThE PERSONALITY AgENT-BASED MODEL}

Based on Lim and Bentley's agent-based model [3] , our model has the following key abstractions:

- Problem. We abstract the shared goal of all agents as the shared task to optimise a function (i.e., find the values of $\boldsymbol{x}$ such that $f(\boldsymbol{x})$ is maximised).

- Individual view of the problem: We model each agent has a choice of either being BAS or BIS activated for each run. If the agent is BAS activated, he will be more motivated to find the maxima and be less concerned with failures. Thus, he will perceive the original team fitness as proportional to $\exp ((1+s) f(\boldsymbol{x}))$, where $s$ is the agent's BAS sensitivity strength. On the other hand, if the agent is BIS activated, he will be more motivated to avoid failures rather than seeking maxima. Hence, he will perceive the original team fitness to be proportional to $\exp (-(1+s) f(\boldsymbol{x}))$, where $s$ is the agent's BIS sensitivity strength.

- Agent psychology. Inspired by swarming algorithms, we model the current mental state of each agent by giving it a position in the solution space (denoting the solution its mind has found so far), a velocity vector (denoting the direction and speed of its thought process), and acceleration vectors (representing the force of ideas and

\footnotetext{
${ }^{1}$ Here 'perception' and 'judgement' is according to Jung's definition where they are not specifically recognised as a separate dimension. 'Perception' is the process of becoming aware (of things), and 'Judgement' is the process of reaching conclusions about what had been perceived. Myers extends on Jung's work to include dimension
}

'Judging vs. Perceiving' where it defines the person's preferred manner of dealing with the outer world. It was proposed in MBTI as a way of determining the dominant and auxiliary functions. 
influences that modify the direction and speed of thought), the latter determined by its personality.

- Agent communication. We model the distribution of information between agents as they each try to solve the same problem. The exact type of information perceived and communicated by each agent and its use is determined by its personality and its BAS/BIS system activated.

Fig. 1 shows the algorithm of the model, and the following sections describe each component in detail.

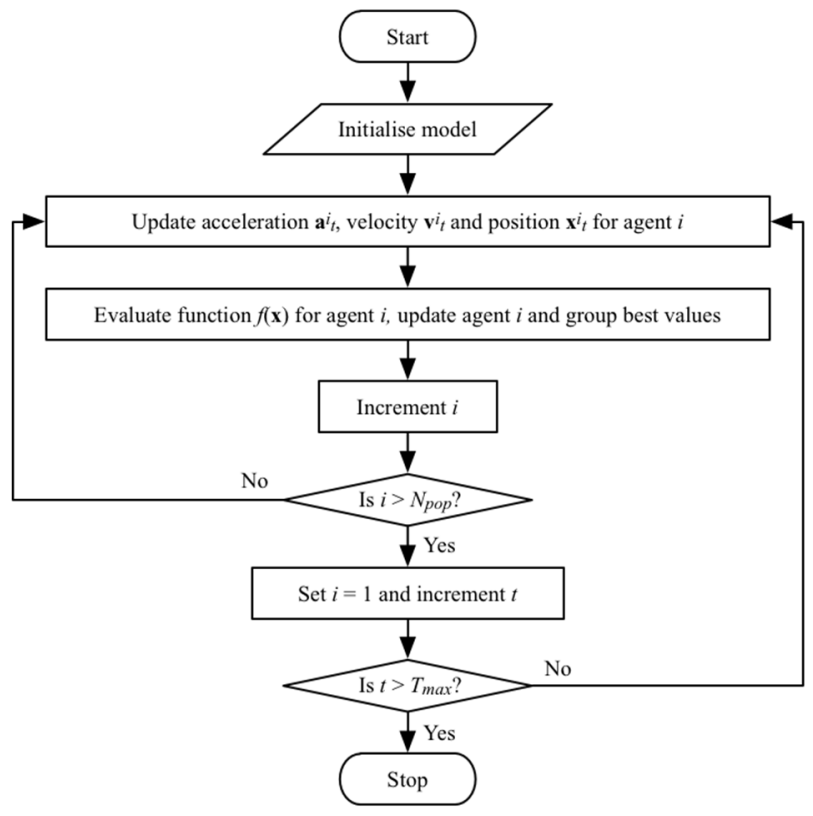

Fig. 1. Algorithm of the model [20].

\section{A. Initialise}

The model is initialised with:

- a problem space $\boldsymbol{D} \in R^{n}$

- an objective function $f(\boldsymbol{x})$

- two BAS/BIS activated objection functions, where $s$ measures the sensitivity towards the corresponding system: $f_{B A S}(\boldsymbol{x}, S), f_{B I S}(\boldsymbol{x}, S): \boldsymbol{D} \rightarrow R$

In particular,

$$
\begin{aligned}
f_{B A S}(\boldsymbol{x}, S) & =\frac{e^{(1+s) f(\boldsymbol{x})}-1}{e^{(1+s)}-1} \\
f_{B I S}(\boldsymbol{x}, s) & =-e^{-(1+s) f(\boldsymbol{x})}+1
\end{aligned}
$$

- the number of timesteps $T_{\max }$ to run the model

- a population of agents $N_{p o p}$, each agent $i \in\left\{1, \ldots, N_{p o p}\right\}$ is initialised with:

○ a personality type $P^{i}$ (one of the MBTI personality types described in the next section)

0 an activated motive system $M^{i}$ (BAS/BIS) and the corresponding sensitivities $s_{M^{i}}$

$\circ$ a position $\boldsymbol{x}_{0}^{i} \in \boldsymbol{D}: \boldsymbol{x}_{\min } \leqslant \boldsymbol{x}_{0}^{i} \leqslant \boldsymbol{x}_{\max }$

$\circ$ a random velocity $\boldsymbol{v}_{0}^{i} \in R^{n}:-\boldsymbol{v}_{\text {init }} \leqslant \boldsymbol{v}_{0}^{i} \leqslant \boldsymbol{v}_{\text {init }}$ $\circ$ personal best (i.e. how the agent perceives dependent on BAS/BIS system) $f_{\text {best }}^{i}=f_{M^{i}}\left(\boldsymbol{x}_{0,}^{i}, S_{M^{i}}\right)$, real personal best (i.e. how the agent actually performs evaluated at original task function) $f^{i}{ }_{\text {realbest }}=f\left(\boldsymbol{x}^{i}{ }_{0}\right)$, personal best position $\boldsymbol{x}_{\text {best }}^{i}=\boldsymbol{x}_{0}^{i}$ and real personal best position $\boldsymbol{x}_{\text {realbest }}^{i}=\boldsymbol{x}_{0}^{i}$

- group best $f^{g}{ }_{\text {best }}$ is the best $f_{\text {realbest }}^{i}$, and group best position $\boldsymbol{x}^{g}$ best ${ }^{g}$ is the corresponding $\boldsymbol{x}_{\text {realbest }}^{i}$

\section{B. Update}

For each timestep $t \in\left\{1, \ldots, T_{\max }\right\}$, each agent $i$ 's position $\boldsymbol{x}^{i}$ is updated using (3):

$$
\boldsymbol{x}^{i}=\boldsymbol{x}_{t-1}^{i}+\boldsymbol{v}^{i}
$$

with the velocity $\boldsymbol{v}_{t}{ }_{t}$ calculated using (4):

$$
\boldsymbol{v}_{t}^{i}=\boldsymbol{v}_{t-1}^{i}+\boldsymbol{a}^{i}
$$

If $\left|\boldsymbol{v}^{i}{ }_{t}\right|>\boldsymbol{v}_{\max }$, it is scaled to equal $\boldsymbol{v}_{\max }$, in order to prevent excessive speed (an individual with high velocity would literally become too "set in their ways" and would find it impossible to change its direction of thought into a useful direction).

Acceleration $\boldsymbol{a}_{t}^{i}$ is used to change the direction and speed of thought, as determined by the agent's personality - one of the 16 MBTI personality types [12]. The interpretation provided here is designed to enable each personality to have an equally good chance of finding the solution. Interpretations were created to represent MBTI personality types appropriately and were not tuned in order to achieve any specific result in later experiments.

Each MBTI personality type has a dominant (more developed) Jungian function supported by an auxiliary (less developed) Jungian function. Here Jungian function inherits the definitions from Jung's work where it looks at a person's general attitude, perceiving and judging functions. For details on how we modeled Jungian functions and the composition of each personality type in dominant and auxiliary Jungian functions, see [3].

Acceleration $\boldsymbol{a}_{t}{ }_{t}$ is calculated using (5):

$$
\boldsymbol{a}^{i}{ }_{t}=\boldsymbol{a}_{J}^{i}+\boldsymbol{a}_{P}{ }^{i}
$$

where $\boldsymbol{a}_{J}{ }^{i}$ is the judging acceleration calculated using Table I and $\boldsymbol{a}_{P}{ }^{i}{ }_{t}$ is the perceiving acceleration calculated using (6):

$$
\boldsymbol{a}_{P}{ }^{i}=\sum_{j=1}^{3} r_{j}\left(\boldsymbol{c}_{j}-\boldsymbol{x}^{i}{ }_{t-1}\right)
$$

where $r_{1}=0.5, r_{2}=0.3$, and $r_{3}=0.2$, and $\boldsymbol{c}_{1}, \boldsymbol{c}_{2}$ and $\boldsymbol{c}_{3}$ are the top 3 candidates derived using

Table II with $f_{M^{l}}\left(\boldsymbol{c}_{1}, s_{M^{l}}\right) \geq f_{M^{2}}\left(\boldsymbol{c}_{2}, s_{M^{2}}\right) \geq f_{M^{3}}\left(\boldsymbol{c}_{3}, s_{M^{3}}\right)$. Here $r_{i}$ 's are the corresponding weights for the top 3 candidates, with decreasing weights as the candidates decrease in their corresponding fitness values. In both tables, agent $i$ 's neighbours are defined as the five nearest agents to agent $i$ measured by Euclidean distance, i.e., the peer group of each agent comprises those who share similar ideas to the agent. To ensure that the 
auxiliary component plays a lesser role compared to the dominant component, $\boldsymbol{a}_{J_{t}}^{i}$ is scaled down such that $\left|\boldsymbol{a}_{J}{ }_{t}{ }_{t}\right|^{2}=\frac{\left|\boldsymbol{a}_{P}{ }_{t}\right|^{2}}{2}$ if $\left.\left|\boldsymbol{a}_{J}^{i}\right|_{t}\right|^{2}>\frac{\mid \boldsymbol{a}_{P}{ }_{t}{ }^{2}}{2}$ (if $P^{i}$ has dominant perception and auxiliary judgment, otherwise vice versa).

\section{Evaluate}

Agent $i$ 's BAS/BIS activated fitness at timestep $t$ is evaluated as $f_{M^{i}, t}^{i}=f_{M^{i}}\left(\boldsymbol{x}^{i}{ }_{t}, s_{M^{i}}\right)$. Finally, the agent's personal best $f^{i}{ }_{\text {best }}$, the agent's real personal best $f^{i}$ realbest , the agent's personal best position $\boldsymbol{x}_{\text {best }}^{i}$, the agent's real personal best position $\boldsymbol{x}_{\text {realbest }}^{i}$, group best $f^{g}{ }_{\text {best }}$ and group best position $\boldsymbol{x}_{\text {best }}^{g}$ are updated.

\section{Settings and Function}

The model was initialised with constant settings in Table III and an objective function $f(\boldsymbol{x})$ as described in (15):

$$
f(x, y)=-\sqrt{x^{2}+y^{2}}
$$

The function was normalised such that $f(x) \in$ [0,1]: $\forall x \in\left[x_{\min }, x_{\max }\right]$. Fig. 2 shows the heatmap and surface plot for original, BAS and BIS activated fitness functions where sensitivities are both 1 . The function represents a simple problem with a clear gradient.

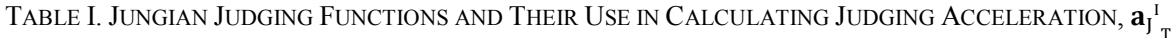

\begin{tabular}{|c|c|}
\hline Function & Implementation \\
\hline $\begin{array}{l}\text { Ti: The agent focusses on its own personal best (the } \\
\text { outcome of its own thoughts). It accelerates towards its } \\
\text { own personal best, with randomness added to enable } \\
\text { exploration. }\end{array}$ & $\begin{array}{l}\boldsymbol{a}_{T i}^{i}{ }_{t}^{i}=\left(\boldsymbol{x}_{{ }_{\text {best }} t-1}-\boldsymbol{x}_{t-1}^{i}\right)+\varphi \\
\text { where } \boldsymbol{x}_{\text {best }}{ }_{t-1} \text { is agent } i \text { 's personal best position in the previous timestep, } \boldsymbol{x}_{t-1}^{i} \text { is the agent's } \\
\text { position in the previous timestep, and } \varphi \text { is a random float in the interval }[-2.0,2.0] .\end{array}$ \\
\hline $\begin{array}{l}\text { Fi: The agent "empathises with" its neighbours' ideas by } \\
\text { accelerating towards its neighbours' average position from } \\
\text { the previous timestep. It also cares about its own personal } \\
\text { thoughts, so accelerates towards its own best position. }\end{array}$ & $\begin{array}{l}\boldsymbol{a}_{F i}{ }_{t}^{i}=\omega_{I} \cdot\left(\mathbb{C}_{n_{t-1}}^{i}-\boldsymbol{x}_{t-1}^{i}\right)+\omega_{2} \cdot\left(\boldsymbol{x}_{\left.{ }_{\text {best }}{ }_{t-1}-\boldsymbol{x}_{t-1}^{i}\right)}\right) \\
\text { where weights } \omega_{I}=0.8, \omega_{2}=0.2, \mathbb{C}_{n}{ }^{i} t-1 \\
\text { neighbours' positions in the previous timestep. }\end{array}$ \\
\hline
\end{tabular}

Table II. Jungian Perceiving Functions and Their Use in Getting Candidates. The First Three Candidates are Returned as $c_{1}, c_{2}$ And $c_{3}$

\begin{tabular}{|c|c|}
\hline Function & Implementation \\
\hline $\begin{array}{l}\text { Si: The agent remembers all its own previous } \\
\text { positions and a few nearby points and their quality. } \\
\text { Candidates are the agent's previous path and new } \\
\text { points near to their position. }\end{array}$ & 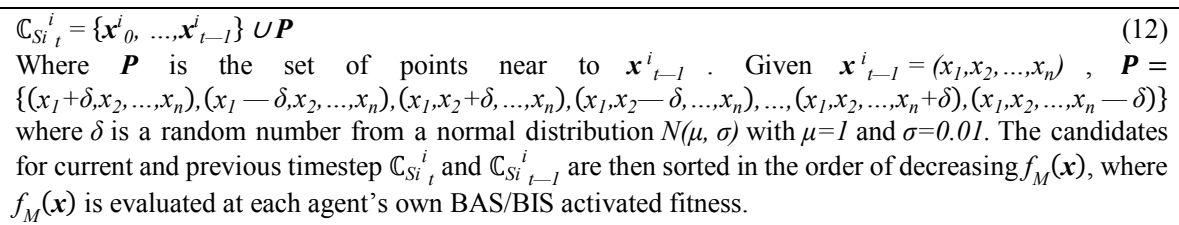 \\
\hline $\begin{array}{l}\text { Ne: The agent sees its neighbours' positions and uses } \\
\text { them to create an "imaginary solution space". } \\
\text { Candidates produced from Se (data from the } \\
\text { environment) are used as input to train the Gaussian } \\
\text { process regression function. Candidates are then the } \\
\text { best quality solutions resulting from sampling this } \\
\text { imaginary space. }\end{array}$ & $\begin{array}{l}f^{*}=G P: \operatorname{train}\left(\mathbb{C}_{S e}, f_{M}\left(\mathbb{C}_{S e}\right)\right) ; \text { predict }\left(\mathbb{C}_{N e}{ }_{t}^{i}\right) \\
\text { where } G P \text { is the Gaussian process regression function [21], training on } \mathbb{C}_{S e} \text { and } f_{M}\left(\mathbb{C}_{S e}\right) \text {, and } \mathbb{C}_{N e}{ }_{t}{ }_{t} \text { is a } \\
\text { vector of points in D. Note } f_{M}(\boldsymbol{x}) \text { is evaluated at each agent's own BAS/BIS activated fitness. The } \\
\text { candidates for current and previous timestep } \mathbb{C}_{N e}{ }_{t}^{i} \text { and } \mathbb{C}_{N e}^{i} e_{t-1} \text { are then sorted in the order of decreasing } \\
f^{*} .\end{array}$ \\
\hline
\end{tabular}


TABLE III. CONSTANTS SETtingS FOR THE MODEL

\begin{tabular}{|c|c|c|c|c|c|c|}
\hline Constants & $T_{\max }$ & $N_{\text {pop }}$ & $\mathbf{v}_{\max }$ & $\mathbf{x}_{\min }$ & $\mathbf{x}_{\max }$ & $\mathbf{v}_{\text {init }}$ \\
\hline Values & 50 & 8 & 5.0 & $(-100,-100)$ & $(100,100)$ & $(1.0,1.0)$ \\
\hline
\end{tabular}
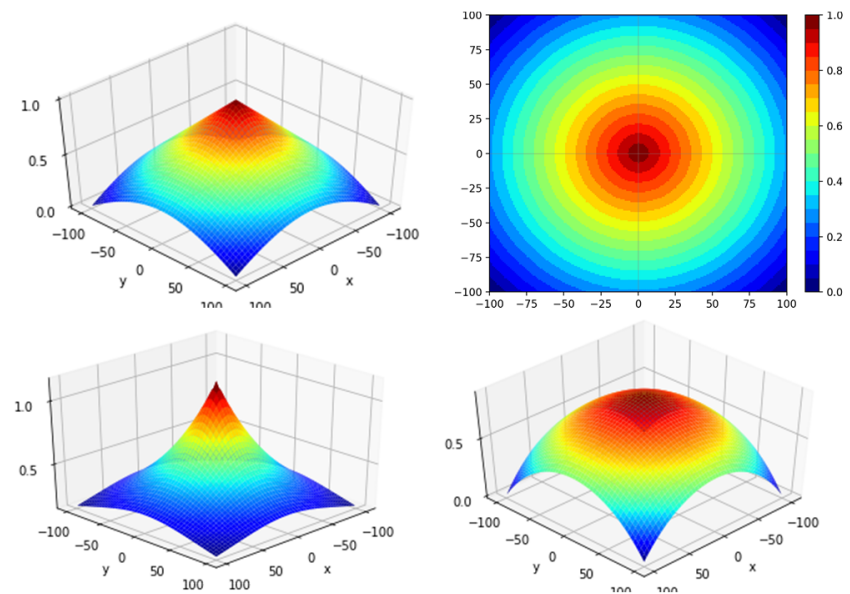

Fig. 2. Surface plot for original fitness (top left) and heatmap (top right) for normalised $(15)$ with a maximum in $(0,0)$. Surface plot for BAS activated fitness (bottom left) and BIS activated fitness (bottom right) with sensitivities $s=1$ under both systems. Colour ranges from blue (minimum) to red (maximum).
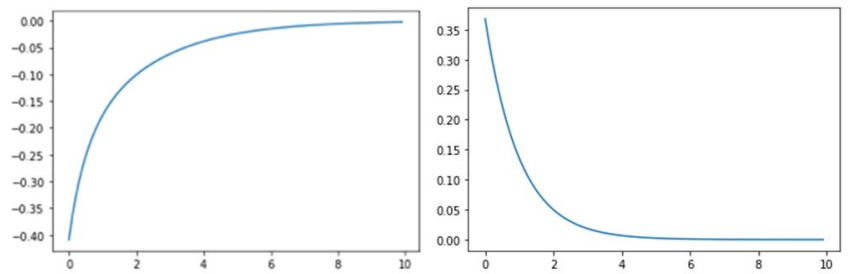

Fig. 3. Derivative of BAS activated objective function with respect to $s$ (left) and derivative of BIS activated objective function with respect to $s$ (right) where $s$ is at $\mathrm{x}$-axis and derivative value at $\mathrm{y}$-axis at $f=1$

\section{E. Adding BAS/BIS to the Agent-based Model}

When the team is working towards a shared goal, each agent will interpret the original task in terms of which system is being activated (BAS/BIS) and their sensitivities. When a BAS activated agent receives signals of reward (i.e., informing them that they are at a good position) there will be more intrinsic motivation to move towards the goal (i.e., finding the maxima) because if they move slightly in the right direction, there will be more gain than normal. On the other hand, when a BAS activated agent receives signals of punishment (i.e., realising that they are at a disadvantageous position) they will be less sensitive to failures since moving towards a worse position will not hurt them as much (and conversely for BIS activated agents).

Modelling using an exponential function guarantees that the BAS activated agent has a reward slope that increases exponentially, and the BIS activated agent has a more gradual reward slope as $f$ increases, as shown in their derivatives (16, 17):

$$
\frac{\partial f_{B A S}(f, s)}{\partial f}=\frac{(1+s) e^{(1+s) f(x)}}{e^{(1+s)}-1}
$$

$$
\frac{\partial f_{B I S}(f, s)}{\partial f}=(1+s) e^{-(1+s) f(x)}
$$

where $s$ is the measure of individual sensitivity to BAS/BIS systems: with increased $s$, sensitivity increases.

We observe from Fig. 3 that as $s$ increases, with fixed $f, f_{B A S}$ increases, and the increased trend decreases when $s$ is already large. Similarly, we can observe that as $s$ increases, with fixed $f, f_{B I S}$ decreases and the decreasing trend decreases when $s$ is already large. This corresponds to the understanding there is a limit to the sensitivity of people to rewards/punishments.

When $s=0$, it is natural to assume that the motive activated fitness is close to the original fitness function. Note, $f_{B A S / B I S}(f, s)=f+O\left(f^{2}\right)$ where we ignore higher order terms as normalised $f$ is within the range $[0,1]$. We again normalise the BAS/BIS activated fitness function to be within the range $[0,1]$.

\section{EXPERIMENTS}

We focus on whether reward sensitivity affects team performance as significantly as the effects of personalities on team performance for uncertain tasks. In order to investigate this question, we conducted three experiments:

E1. Does BAS/BIS affect team performance for all personality groups for tasks with and without uncertainty?

E2. Does the BAS/BIS sensitivity strength parameter $s$ affect on team performance?

E3. After adding in BAS/BIS system into the model, can effects of BAS/BIS on team performance overwhelm or counteract the original performance difference caused by differences in personalities?

We created teams with opposing MBTI dichotomies: Extraverts vs. Introverts, Sensors vs. Intuitives, Thinkers vs. Feelers, and Judgers vs. Perceivers. Each team has 8 agents with personality described in Table IV.

Here we compare the control group (i.e., the original model without adding motive activated objective functions [3] ), BAS group (i.e., each agent is BAS activated), BIS group (i.e., each agent is BIS activated). Each experiment was repeated 500 times. Task uncertainty is modelled by adding noise to the objective function [ 3$]$.

TABLE IV. TEAM AND AGENT PERSONALITY

\begin{tabular}{|c|c|c|c|c|c|c|c|c|}
\hline Team & \multicolumn{7}{|c|}{ Agent Personality } \\
\hline Extraverts & ESTP & ESFP & ENFP & ENTP & ESTJ & ESFJ & ENFJ & ENTJ \\
\hline Introverts & ISTP & ISFP & INFP & INTP & ISTJ & ISFJ & INFJ & INTJ \\
\hline Sensors & ISTJ & ISFJ & ISTP & ISFP & ESTP & ESFP & ESTJ & ESFJ \\
\hline Intuitives & INFJ & INTJ & INFP & INTP & ENFP & ENTP & ENFJ & ENTJ \\
\hline Thinkers & ISTJ & INTJ & ISTP & INTP & ESTP & ENTP & ESTJ & ENTJ \\
\hline Feelers & ISFJ & INFJ & ISFP & INFP & ESFP & ENFP & ESFJ & ENFJ \\
\hline Judgers & ISTJ & ISFJ & INFJ & INTJ & ESTJ & ESFJ & ENFJ & ENTJ \\
\hline Perceivers & ISTP & ISFP & INFP & INTP & ESTP & ESFP & ENFP & ENTP \\
\hline
\end{tabular}




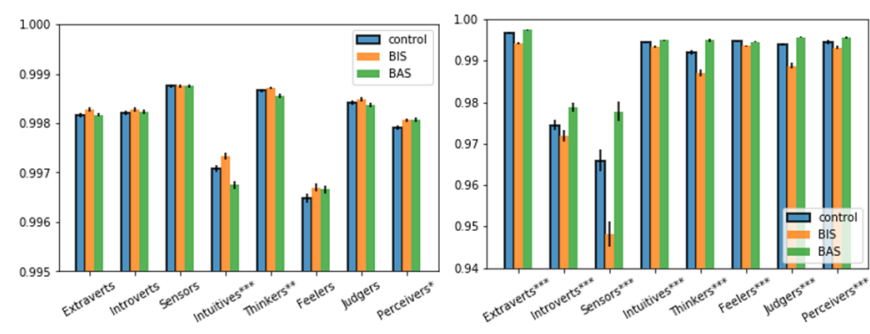

Fig. 4. E1 Teams with control, BAS and BIS groups under no noise in task(left) and under $20 \%$ noise in task(right): average group performance (y-axis) over personality teams (x-axis). Error bars represent one standard error. * indicates that the ANOVA results show a significant difference among the averages for the BIS/BAS activated group and control group: * at $p<.05, * *$ at $p<.01$ and $* * *$ at $p<.001$.

\section{RESULTS}

\section{A. Experiment E1: BAS/BIS effect on Team Performance for task with and without uncertainty}

Fig. 4. provides the results for experiment E1 under no noise and under $20 \%$ noise. We compared the average group best for control, BAS, BIS groups under 8 personality groups where each agent being BAS/BIS activated has sensitivity 1 . In order to investigate whether BAS/BIS systems have an effect on performance, we use ANOVA to check if they have significantly different means. We can see 5 out of 8 personality groups do not have significant difference between BAS, BIS, control system in terms of performance in tasks without uncertainty. There are 3 personality groups that have significant difference in group performance: Intuitives, Thinkers and Perceivers. Though they are statistically significant, we can see the range of performance differences for those statistically significant groups has mean range 0.0003 , which is relatively small compared to $20 \%$ noise setting. In general, under no noise setting, the mean range of performance differences is 0.00017 ; when there is high noise, on the other hand, we observe all personality groups have statistically significant difference in means between BAS/BIS and control groups with $p<.001$. Moreover, the mean range of performance differences for all personality groups with uncertainty is 0.0074 - far larger than that without uncertainty. This indicates that for majority of personality groups on tasks with low uncertainty, BAS/BIS have little effect; BAS/BIS have significant effect on all personalities for performance tasks with high uncertainty.

\section{B. Experiment E2: BAS/BIS sensitivities on Team Performance}

Next, to investigate the properties of our augmented model, we test how different levels of BAS/BIS sensitivities influence group performance. Following the results from E1, we focus on high uncertainty (i.e. $20 \%$ noise in task). Fig. 5 and 6 provide the results for experiment E2 under BAS and BIS systems respectively. We differentiate the control group in the original model (left most bar with bold outlines) with our augmented model under different sensitivities (i.e. $s$ takes values in range of $[0,1,2,4,6,8,10])$.
We perform ANOVA on sensitivities ranging from 0 to 10 (excluding the control group) and we observe that for BAS system, Extraverts, Sensors, Thinkers, Feelers and Judgers' performances are significantly influenced overall by different sensitivities; for BIS system, all personality groups are significantly influenced overall by different sensitivity levels. Very interestingly, we observe as BIS sensitivity increases, for all personality groups, they show a decreasing trend in performance; As BAS sensitivity increases, we observe performance increases initially but starts decreasing or flattening when $s$ becomes too large.

In summary, all levels of BIS sensitivity significantly decrease team performance for all personality groups; however only low levels of BAS sensitivity significantly increase team performance for majority of personality groups.

\section{Experiment E3: Differences in personality performance comparisons with BAS/BIS addition to model}

In comparison to the original model []ㅡ, we investigate how adding in BAS/BIS system will change the original differences observed in team performance based on personalities for uncertain tasks (i.e. can they overwhelm or counteract the performance differences caused by personality?). Fig. 7 provides the results for E3. We compared the opposing MBTI dichotomies: Extraverts (E) vs. Introverts (I), Sensors (S) vs. Intuitives (N), Thinkers (T) vs. Feelers (F), Judgers (J) vs. Perceivers (P). Fig. 7 (top) shows the personality team performance in original model [3]; Fig. 7 (bottom left) compares the original outperforming personality being BIS activated with the original underperforming personality; Fig. 7 (bottom right) compares the original underperforming personality being BAS activated with the original outperforming personality.

For Extraverts vs. Introverts, we observe that being BAS activated increases Introverts' performance and being BIS activated decreases Extraverts' performance, however there is no change in the fact that Extraverts outperform Introverts in tasks of uncertainty. For Sensors vs. Intuitives, we observe that being BAS/BIS influenced still did not counteract the original personality advantage: Intuitives still outperform Sensors even when Intuitives are BIS activated or Sensors are BAS activated. For both Thinkers vs. Feelers, Judgers vs. Perceivers groups, the group means after BIS/BAS activated are very close to each other and it seems that BAS/BIS effects may have counteracted the performance difference caused by personalities. In order to assess whether they are indeed statistically equivalent, we perform equivalence test under $95 \%$ confidence interval. We let the equivalence margin be the mean difference between the control group and BAS/BIS activated group with sensitivity 0 , then perform two one-sided tests (TOST). Table V shows the results. We observe that Thinkers vs. Feelers group is tested to be equivalent with statistical significance. This indicates that Thinkers with BAS activated are indeed catching up despite the original personality differences; similarly, Feelers with BIS activated can counteract the original advantage of personality in performance. 

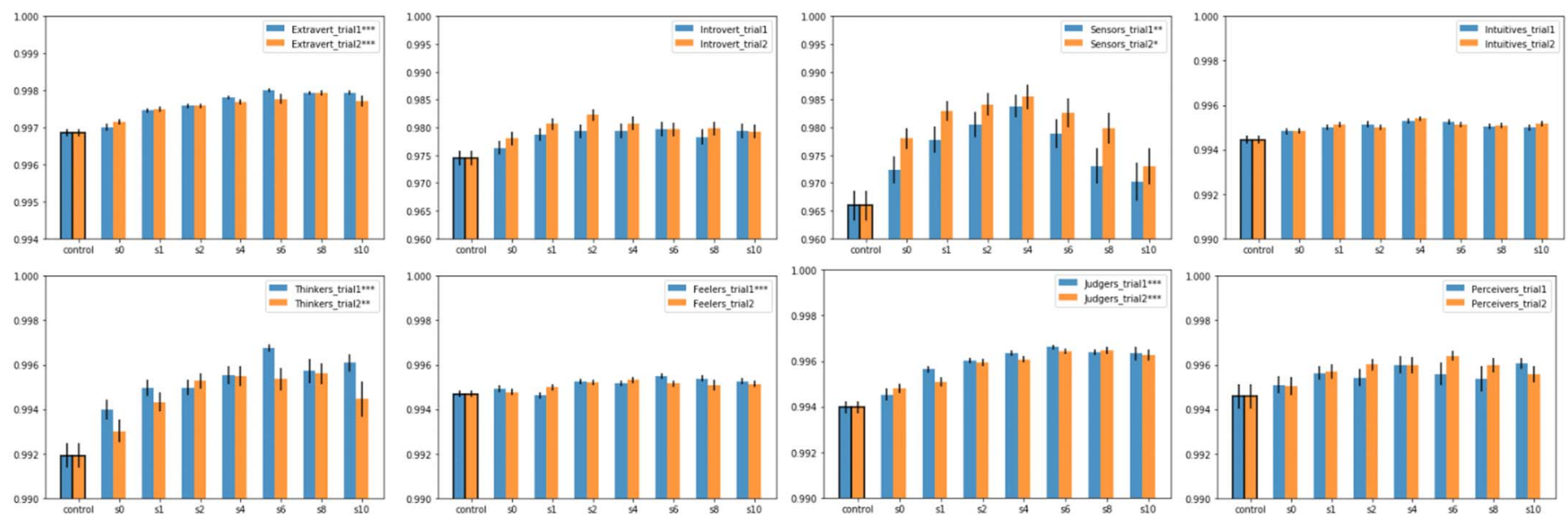

Fig. 5. Experiment 2 with $20 \%$ noise: average group performance (y-axis) over BAS sensitivities (x-axis) for 8 different personality teams. Error bars represent one standard error. * indicates that the ANOVA results show a significant difference for different BAS sensitivities on group performance: $*$ at $\mathrm{p}<.05, * *$ at $\mathrm{p}<.01$ and $* * *$ at $\mathrm{p}<.001$.
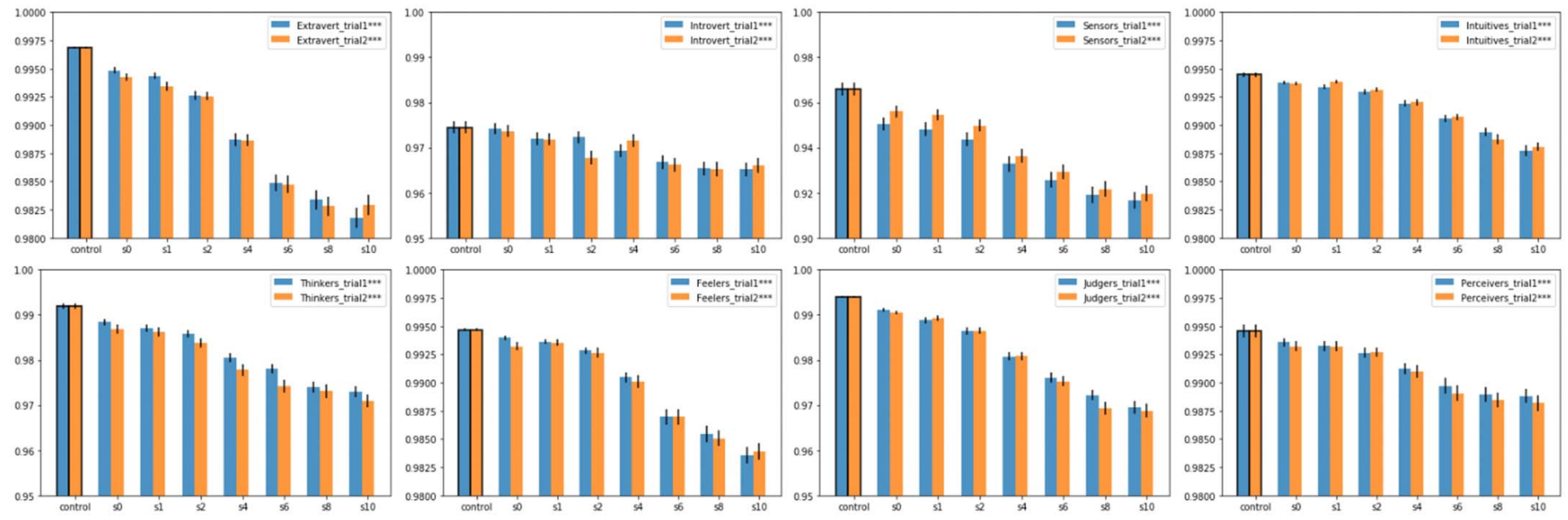

Fig. 6. Experiment 2 with $20 \%$ noise: average group performance (y-axis) over BIS sensitivities (x-axis) for 8 different personality teams. Error bars represent one standard error. * indicates that the ANOVA results show a significant difference for different BIS sensitivities on group performance: * at $p<.05, * *$ at $p<.01$ and *** at $p<.001$.

TABLe V. Equivalence TeSt Significance

\begin{tabular}{|l|c|c|c|}
\hline $\begin{array}{l}\text { Null hypothesis } \\
\left|\boldsymbol{\mu}_{\mathbf{1}}-\boldsymbol{\mu}_{\mathbf{0}}\right|>\boldsymbol{\delta}\end{array}$ & $\begin{array}{l}\text { Mean } \\
\text { difference } \\
\boldsymbol{\mu}_{\mathbf{0}}-\boldsymbol{\mu}_{\mathbf{1}}\end{array}$ & $\begin{array}{l}\text { Acceptance } \\
\text { range }(\boldsymbol{\delta})\end{array}$ & $\begin{array}{l}\text { Equivalence } \\
\text { with 95\% } \\
\text { confidence }\end{array}$ \\
\hline $\begin{array}{l}\mu_{0}: \text { Introvert Control } \\
\mu_{1}: \text { Extravert BIS }\end{array}$ & -0.018 & 0.0011 & False \\
\hline $\begin{array}{l}\mu_{0}: \text { Extravert Control } \\
\mu_{1}: \text { Introvert BAS }\end{array}$ & 0.018 & 0.0011 & False \\
\hline $\begin{array}{l}\mu_{0}: \text { Sensor Control } \\
\mu_{1}: \text { Intuitive BIS }\end{array}$ & -0.027 & 0.011 & False \\
\hline $\begin{array}{l}\mu_{0}: \text { Intuitive Control } \\
\mu_{1}: \text { Sensor BAS }\end{array}$ & 0.014 & 0.011 & False \\
\hline $\begin{array}{l}\mu_{0}: \text { Thinker Control } \\
\mu_{1}: \text { Feeler BIS }\end{array}$ & -0.00091 & 0.0028 & True \\
\hline $\begin{array}{l}\mu_{0}: \text { Feeler Control } \\
\mu_{1}: \text { Thinker BAS }\end{array}$ & -0.0002 & 0.0028 & True \\
\hline $\begin{array}{l}\mu_{0}: \text { Perceiver Control } \\
\mu_{1}: \text { Judger BIS }\end{array}$ & 0.0013 & 0.0017 & False \\
\hline $\begin{array}{l}\mu_{0}: \text { Judger Control } \\
\mu_{1}: \text { :erceiver BAS }\end{array}$ & -0.0014 & 0.0017 & False \\
\hline
\end{tabular}
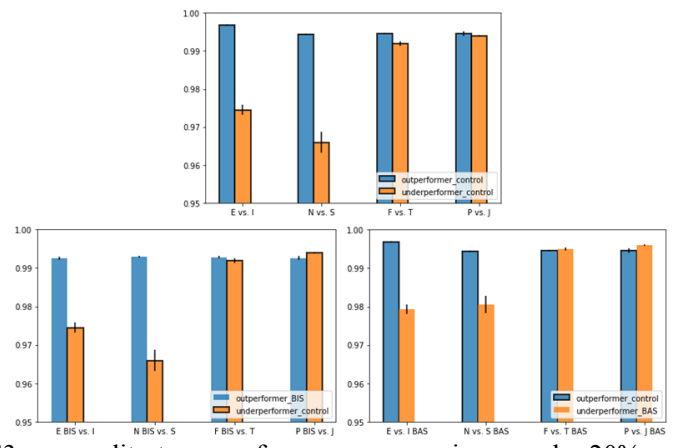

Fig. 7. E3 personality team performance comparisons under $20 \%$ noise: (top) control group represents performance in original model, (bottom left) BIS group represents the original outperforming personality being BIS activated for sensitivity 2 compared to original underperforming personality, (bottom right) BAS group represents the original underperforming personality being BAS activated with sensitivity 2 compared to original outperforming personality. Error bars represent one standard error. 


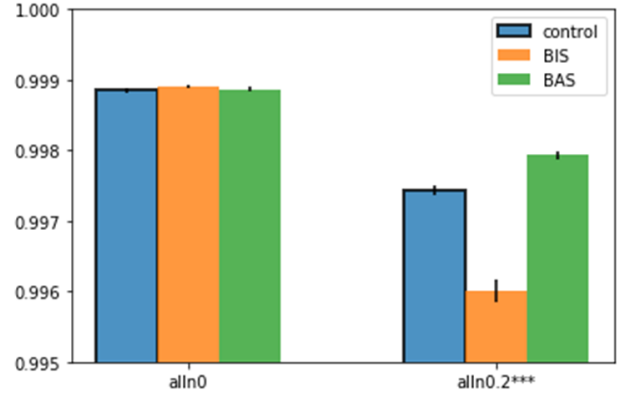

Fig. 8. All personality in one team for no noise (left) and $20 \%$ noise (right): average group performance (y-axis) for 3 different groups (control, BAS activated, BIS activated). Error bars represent one standard error. * indicates that the ANOVA results show a significant difference among three groups on performance: * at $p<.05,{ }^{* *}$ at $p<.01$ and $* * *$ at $p<.001$.

\section{ANALYSIS}

Following the experiments, the findings were assessed to see whether the predictions made by the model were supported by the literature.

Jackson et al. [ 8$]$ examined the relationship between executive functioning and Gray's original RST theory. The participants completed objective tests, which are tasks with no noise. They found no evidence in BIS will be negatively related to executive functioning performance and only very minor support of BAS will be positively related to executive functioning performance.

Franken and Muris [9] examined the relationship between performance on decision making and Gray's Theory. The participants completed the IOWA gambling task where they try to maximise profit by drawing card from one of four different decks - a task with uncertainty. The results showed that good performance on IOWA gambling task was found positively associated to BAS, but not to BIS.

Hutchison, Burch and Boxall [10] tested the links between senior-executive job performance and the Gray's revised reinforcement sensitivity theory. The results showed that BAS has no main effect and BIS has marginally significant effect on performance.

The first two works corroborate our model predictions that BAS/BIS systems significantly influence team performance in tasks with uncertainty, and not in tasks with no noise. The result is shown in Fig. 8. assuming participants are randomly distributed among 16 personalities. The third work supports our prediction that BIS has significant effect in team performance for tasks of uncertainty.

\section{CONCLUSION}

In this work we added Gray's reinforcement sensitivity theory to the original agent-based modelling for MBTI personality types in order to explore the combined effect on team performance for a more complex and closer to reality personality structure. As discovered by our new model, for uncertain tasks, the behavioural activation system and behavioural inhibition system significantly influenced team performance at tasks of uncertainty. The model predicted that a majority of personality teams are significantly influenced by behavioural activation system, and this finding is corroborated by real-world studies. The performance is nonlinear as sensitivity increases. The model also predicted that all personality teams are significantly negatively influenced by behavioural inhibition system. The more sensitive to punishments, the worse teams perform.

\section{REFERENCES}

[1] J. A. Gray, "The psychophysiological basis of introversion-extraversion: A modification of Eysenck's theory," in The biological bases of individual behaviour: Academic Press, 1972.

[2] J. A. Gray, "A critique of Eysenck's theory of personality," A model of personality, pp. 246-276, 1981.

[3] S. L. Lim and P. J. Bentley, "Coping with Uncertainty: Modelling Personality when Collaborating on Noisy Problems," in Artificial Life Conference Proceedings, 2018, pp. 566-573.

[4] N. Schoots, S. L. Lim, and P. J. Bentley, "Investigating Conformity by Personality Type," in Proceedings of the 2019 International Workshop on Agent-Based Modelling of Human Behaviour (ABMHuB), 2019.

[5] S. L. Lim and P. J. Bentley, "All in Good Team: Optimising Team Personalities for Different Dynamic Problems and Task Types," in Artificial Life Conference Proceedings, 2019, pp. 153-160.

[6] S. L. Lim and P. J. Bentley, "Diversity Improves Teamwork: Optimising Teams using a Genetic Algorithm," 2019 IEEE Congress on Evolutionary Computation (CEC), pp. 2848-2855, 2019.

[7] C. Jung, Psychological Types. Oxford, England: Harcourt, Brace, 1923.

[8] C. J. Jackson, N. J. Loxton, P. Harnett, J. Ciarrochi, and M. J. Gullo, "Original and revised reinforcement sensitivity theory in the prediction of executive functioning: A test of relationships between dual systems," Personality and Individual Differences, vol. 56, pp. 83-88, 2014.

[9] I. A. Franken and P. Muris, "Individual differences in decisioin-making," Personality and Individual Differences, vol. 39, pp. 991-998, 2005.

[10] A. Hutchison, G. Burch, and P. Boxall, "Reinforcement sensitivity theory as a predictor of senior-executive performance," Personality and Individual Differences, vol. 55, pp. 931-935, Nov 2013.

[11] L. R. Goldberg, "An alternative" description of personality": the big-five factor structure," Journal of Personality and Social Psychology, vol. 59, p. $1216,1990$.

[12] I. B. Myers, "The Myers-Briggs Type Indicator: Manual (1962)," 1962.

[13] H. J. Eysenck, The biological basis of personality. Springfield, Ill.: Charles C. Thomas, 1967.

[14] J. Salvit and E. Sklar, "Modulating agent behavior using human personality type," in Proceedings of the Workshop on Human-Agent Interaction Design and Models (HAIDM) at Autonomous Agents and MultiAgent Systems (AAMAS), 2012, pp. 145-160.

[15] A. Campos, F. Dignum, V. Dignum, A. Signoretti, A. Magály, and S. Fialho, "A process-oriented approach to model agent personality," in Proceedings of The 8th International Conference on Autonomous Agents and Multiagent Systems-Volume 2, 2009, pp. 1141-1142.

[16] S. Ahrndt, J. Fähndrich, and S. Albayrak, "Modelling of personality in agents: from psychology to implementation," Proceedings of the HAIDM, pp. 1-16, 2015.

[17] F. Durupinar, N. Pelechano, J. Allbeck, U. Gudukbay, and N. I. Badler, "How the ocean personality model affects the perception of crowds," IEEE Computer Graphics and Applications, vol. 31, pp. 22-31, 2011.

[18] C. S. Carver and T. L. White, "Behavioral Inhibition, Behavioral Activation, and Affective Responses to Impending Reward and Punishment: The BIS/BAS Scales," Journal of Personality and Social Psychology, vol. 67, pp. 319-333, 1994.

[19] J. A. Gray, The psychology of fear and stress. 2nd ed ed. Cambridge: Cambridge University Press, 1987.

[20] S. Ahrndt, A. Aria, J. Fähndrich, and S. Albayrak, "Ants in the OCEAN: Modulating agents with personality for planning with humans," in European Conference on Multi-Agent Systems, 2014, pp. 3-18.

[21] C. K. Williams and C. E. Rasmussen, "Gaussian processes for regression," in Advances in Neural Information Processing Systems, 1996, pp. $514-520$. 